\title{
Nomenclatura anatômica em oftalmologia
}

\author{
Anatomical nomenclature in ophthalmology
}

\author{
Ricardo Lamy ${ }^{1}$ \\ Adalmir Morterá Dantas²
}

\begin{tabular}{|l|}
\hline RESUMO \\
\hline Os objetivos deste estudo são: informar os oftalmologistas sobre as \\
diferenças existentes entre as listas em língua inglesa e portuguesa de \\
termos equivalentes para as estruturas do olho, ambas aprovadas pela \\
Comissão Federativa Internacional de Terminologia Anatômica; apresen- \\
tar os termos anatômicos incluídos na lista de descritores publicada pela \\
Biblioteca Nacional de Medicina Norte-Americana e traduzida pela Bi- \\
blioteca Regional de Medicina (BIREME); para propor uma lista em \\
português de termos de uso comum pelos oftalmologistas.
\end{tabular}

Descritores: Terminologia; Anatomia; Olho; Oftalmologia; Descritores em medicina; Epônimos; História da medicina

\section{INTRODUÇ̃̃̃O}

A elevada freqüência com que os oftalmologistas utilizam termos inadequados para designar as estruturas anatômicas do olho, e as diferenças entre os termos reconhecidos como equivalentes da terminologia anatômica latina, em inglês e na língua portuguesa, justificam a realização deste estudo de revisão sobre a história da anatomia e da etimologia dos termos anatômicos, assim como a divulgação das listas de termos equivalentes em inglês, português, e a proposição e apresentação de uma lista contendo os termos de uso corrente na literatura oftalmológica nacional.

$\mathrm{Na}$ história da origem da anatomia, o mais antigo tratado anatômico existente é um papiro egípcio escrito por volta de 1600 a.C $\mathrm{C}^{(1)}$. Ele demonstra que o coração, fígado, baço, rins, ureteres e vesícula já eram conhecidos, assim como o fato de os vasos sanguíneos se originarem no coração. Centenas de anos depois, um professor de anatomia de Pádua chamado Andréas Vesalius (1514-1564) publicava sua monumental obra intitulada De Humani Corporis Fabrica Libri Septem (1543). Ilustrada pelo artista John Calcar, a obra padronizava uma terminologia anatômica e serviu de referência para todo o mundo civilizado à época. Com o passar dos anos, outros centros científicos foram publicando seus próprios livros, onde incluíam novos nomes para estruturas antes desconhecidas e corrigiam os nomes que julgavam impróprios. Desta forma, diferentes nomes foram sendo criados e se acumulando, chegando a existir na Europa no final do século XIX mais de cinqüenta mil nomes para designar estruturas anatômicas. Alguns dicionários médicos listavam mais de vinte sinônimos para uma única estrutura, induzindo a uma enorme confusão ${ }^{(2)}$.

Percebendo a necessidade de se tentar uniformizar a nomenclatura, a Sociedade de Anatomia Alemã iniciou em 1887 (Leipzig) o trabalho de elaboração de uma lista que veio a ser aprovada em 1895 durante o Congresso de Anatomia da Basiléia. A obra ficou conhecida como Basle Nomina Anatomica (BNA) e foi publicada contendo 5.228 termos em latim. No ano 
de 1903 foi fundada a International Federation of Associations of Anatomists (IFAA), que pretendia reunir-se a cada cinco anos, tendo como um de seus objetivos a seleção de uma nomenclatura uniforme e universal para as ciências anatômicas. Nos anos que se seguiram, a Sociedade Britânica de Anatomia e a Sociedade Alemã de Anatomia apresentaram suas próprias atualizações da BNA (Birminghan em 1933; Jena em 1935), mas estas revisões não foram bem aceitas internacionalmente. Em 1950, durante o Congresso Internacional de Oxford, foi designada pelo presidente da IFAA uma Comissão Internacional para Nomenclatura Anatômica (IANC), cuja tarefa principal era preparar uma lista de termos anatômicos que seriam submetidos à aprovação durante o congresso seguinte em Paris. Assim, em 1955 foi aprovada com unanimidade a Parisiensia Nomina Anatomica, contendo 5.640 termos em latim (1.354 nomes de estruturas descobertas após a BNA; os segmentos anátomo-cirúrgicos dos pulmões; o grupo de órgãos endócrinos e nenhum epônimo).

A terceira edição da Nomina foi publicada em 1966 e em 1975 veio a quarta edição, desta vez contendo a Nomina Embryologica e a Nomina Histologica. Após lançamento da quinta edição em 1983, membros do IANC propuseram transformar a comissão em um corpo editorial independente da IFAA e em 1989 publicaram uma sexta edição da Nomina Anatomica sem submeter a lista à aprovação dos membros da IFAA durante o Congresso Internacional do Rio de Janeiro. Foi convocada então uma Assembléia Geral da IFAA, sob a presidência do brasileiro Liberato Di Dio, onde foi aprovada com unanimidade a criação de uma nova Comissão Federativa de Terminologia Anatômica (FCAT) cujos membros seriam eleitos de forma democrática, objetivando representar os anatomistas dos cinco continentes. Em 1997, após o término do Congresso Internacional de São Paulo, o FCAT anunciou oficialmente o lançamento da nova Terminologia Anatomica $(\mathrm{TA})^{(3)}$ simplificada e atualizada, contendo pela primeira vez além dos termos em latim, uma lista com termos de uso corrente em inglês (que embora sejam reconhecidos como termos equivalentes, não são necessariamente as traduções dos termos em latim). Em 1998, a Sociedade Brasileira de Anatomia (SBA) em conjunto com o FCAT, publicam pela editora Manole a edição em língua portuguesa da $\mathrm{TA}^{(4)}$ contendo a lista de termos em latim e uma lista de equivalentes em português. Embora esta lista pudesse incluir termos de uso corrente na língua portuguesa, observa-se, na seção destinada aos órgãos dos sentidos (e em especial nas estruturas relacionadas ao olho), haver apenas uma tradução direta dos termos em latim, diferentemente da lista preparada pelos países de língua inglesa. Uma das poucas exceções é o termo "ducto lacrimonasal", equivalente do termo em latim ductus nasolacrimalis, e cujo equivalente em inglês é nasolacrimal duct.

No ano de 2005, Abib e Oréfice publicaram excelente arti$\mathrm{go}^{(5)}$ na seção de atualização continuada dos Arquivos Brasileiros de Oftalmologia objetivando divulgar à classe oftalmológica a existência da edição em língua portuguesa da TA.
Em trabalho ${ }^{(6)}$ apresentado no XXXIV Congresso Brasileiro de Oftalmologia, Lamy, Dantas et al., avaliam o conhecimento e a aplicação pelos oftalmologistas dos termos aprovados pelo FCAT (agora chamado FICAT: Comissão Federativa Internacional de Terminologia Anatômica) para designar as estruturas anatômicas da córnea. Os autores utilizaram um questionário objetivo de múltipla escolha (Figura 1), com versões em inglês e português, onde o médico participante deveria assinalar os termos anatômicos adequados para designar cada uma das cinco camadas da córnea. O questionário foi distribuído durante o Congresso Mundial de Oftalmologia de São Paulo e respondido por 85 médicos de 26 países (Figura 2). $9,41 \%$ eram residentes de oftalmologia e, dentre os especialistas em oftalmologia, 44,83\% declararam ser sub-especialistas em córnea. Nenhum entrevistado assinalou o termo anatômico correto para todas as cinco camadas da córnea. 90,59\% utilizaram os termos membrana de Bowman e membrana de Descemet para designar a lâmina limitante anterior e a lâmina limitante posterior da córnea. Nenhum participante assinalou o termo epitélio posterior da córnea.

Se considerarmos a lista de termos equivalentes em inglês publicada na TA, poderiam ser considerados corretos os questionários que tiveram o termo endothelium escolhido para designar a camada mais interna da córnea.

Embora esteja claro no prefácio da TA que a lista de termos equivalentes em inglês não deve ser usada como referencial para tradução para outras línguas, lamentamos que a

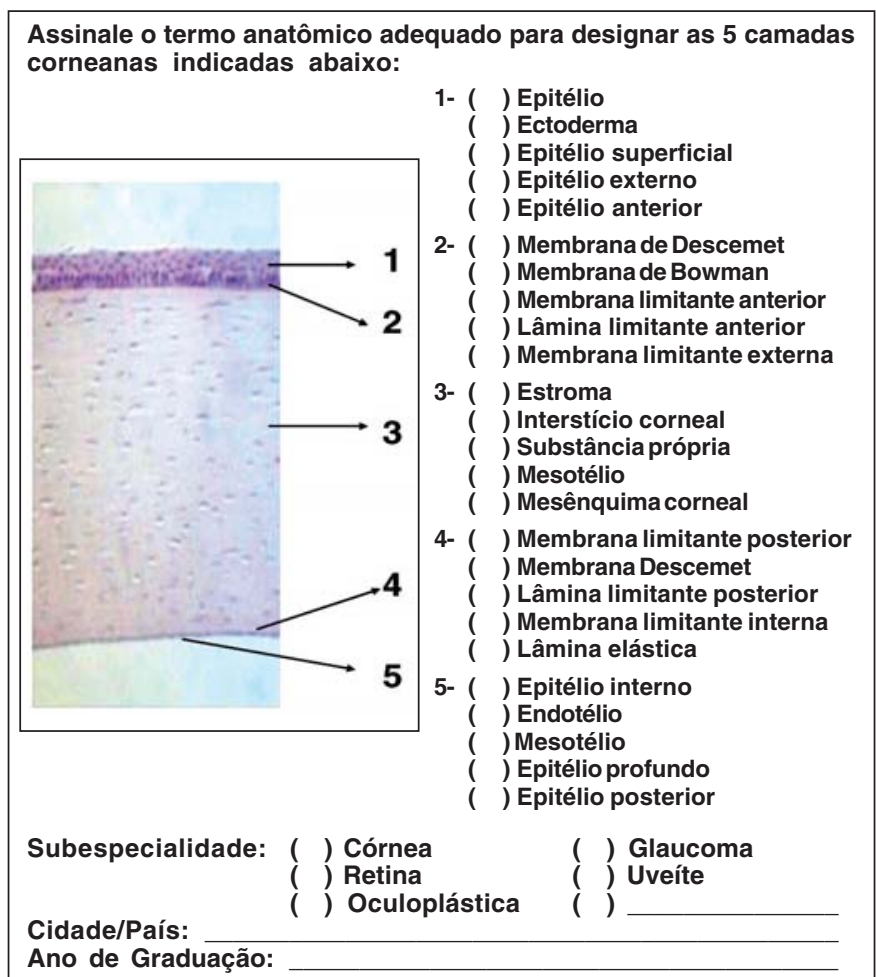

Figura 1 - Questionário utilizado para avaliar o conhecimento e uso da terminologia anatômica na designação das estruturas da córnea (versão em português) ${ }^{(6)}$ 


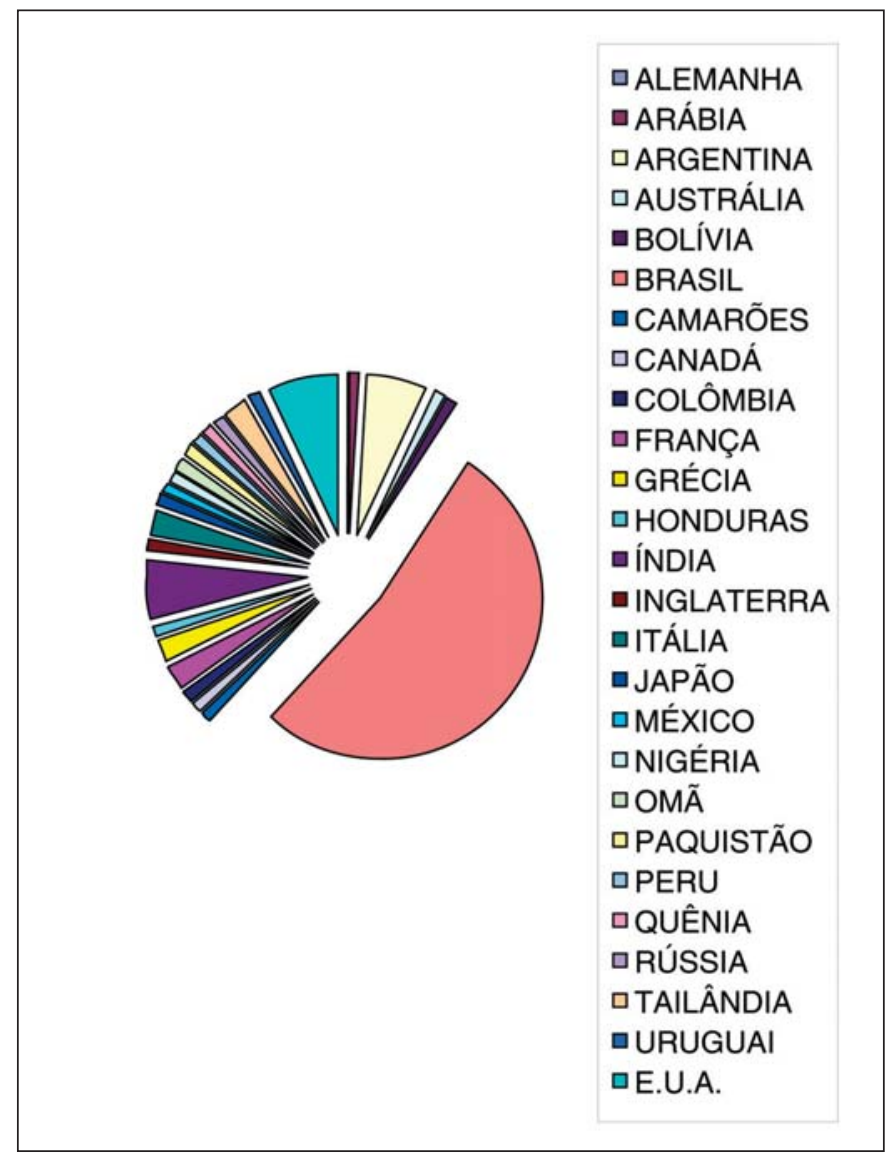

Figura 2 - Nacionalidade dos médicos que responderam ao questionário(6)

seção de estruturas relacionadas ao olho da lista de termos equivalentes em língua portuguesa tenha sido elaborada, aparentemente sem levar em consideração os termos de uso corrente na literatura oftalmológica nacional.

Considerando a importância dos trabalhos desenvolvidos em língua inglesa na literatura médica da atualidade, parecenos claro a iminente confusão quando, por exemplo, é aprovado pelo FICAT o uso do termo endothelium of anterior chamber, enquanto na língua portuguesa, reconhece-se apenas o termo epitélio posterior da córnea. A confusão fica ainda mais evidente quando um trabalho publicado em língua portuguesa é acompanhado por um resumo em inglês (abstract) contendo algum destes termos. Desta forma, propomos uma lista de termos anatômicos oftalmológicos de uso corrente na língua portuguesa (Quadro 1). Esperamos que esta lista possa servir de referência para comunidade oftalmológica brasileira, permitindo o reconhecimento destes termos, ao menos enquanto se aguarda a aprovação de uma segunda edição da TA. Para elaboração desta lista, revisamos diversos livros da literatura médica nacional, em especial os pertencentes à bibliografia mínima $^{(7-26)}$ sugerida pelo Conselho Brasileiro de Oftalmologia para os candidatos ao título de especialista em oftalmologia. Estão presentes nesta lista termos já consagrados no meio oftalmológico, como coróide ao invés de corióide, e tivemos o cuidado de não "criar" nenhum termo novo, que não tenha seu correspondente em inglês incluso na lista de termos equivalentes ou na lista de termos descritores.

Apesar da frequiente utilização dos epônimos na literatura oftalmológica (dezenas de anos após serem banidos da terminologia anatômica), optamos por excluí-los da lista de termos de uso corrente. O uso de epônimos é causa freqüente de confusão, visto que algumas vezes temos descrito mais de um epônimo para uma mesma estrutura anatômica, ou até um único epônimo para duas estruturas anatômicas diferentes (ex: o termo músculo de Muller pode ser utilizado para designar as fibras circulares do músculo ciliar ou o músculo orbital.). Além disso, epônimos são termos inespecíficos e o termo anatômico deve ter uma característica localizadora, descritiva ou etiológica que facilite a compreensão dos fatos ou sua ligação com a natureza ou a causa do assunto em questão(27-28). Contudo, visto que muitos epônimos ainda são aceitos como termos descritores em ferramentas de pesquisa; faz-se necessário o conhecimento de alguns destes termos (epônimos) pelos oftalmologistas. O quadro 2 contém os principais epônimos oftalmológicos seguidos pelos termos anatômicos adequados, aprovados pelo FICAT.

Os Descritores em Ciências da Saúde (DeCS), derivados do termo em língua inglesa Medical Subject Headings (MeSH) são publicados pela Biblioteca Nacional de Medicina Norte Americana (U.S. National Library of Medicine), responsável pela base de dados Medline que é uma das mais completas e utilizadas na pesquisa bibliográfica na área das Ciências da Saúde.

Outras bases de dados como o LILACS (Literatura Médica da América Latina e do Caribe) e SciELO (Scientific Eletronic Library Online) também disponíveis na Biblioteca Regional de Medicina (BIREME) usam a padronização dos DeCS.

É fundamental que os autores dos trabalhos científicos façam uma escolha criteriosa e adequada destes unitermos, para que a indexação permita a recuperação de seus trabalhos, quando procurados pelos seus pares em uma pesquisa bibliográfica. O quadro 3 apresenta a lista de descritores (DeCS) para as estruturas anatômicas do olho, em português e inglês. A coluna do meio apresenta os sinônimos para língua portuguesa indexados pela BIREME. Consideramos indevida a utilização de alguns destes sinônimos, como é o caso do termo rubor aquoso.

A fim de complementar este trabalho, organizamos um vocabulário etimológico de termos anatômicos utilizados em oftalmologia ${ }^{(29-38)}$. O estudo da etimologia é de grande valor mnemônico, pois, desde os tempos remotos, a maioria dos termos foi criada com base em semelhanças com objetos de uso doméstico, com a natureza, a geometria e funções desempenhadas pelos órgãos do corpo.

Esperamos estar contribuindo com todos aqueles que pretendem empregar, de forma correta, os termos anatômicos para as estruturas relacionadas ao olho, elucidando as diferenças existentes entre as listas de termos equivalentes em inglês, português e a lista de termos descritores. 


\begin{tabular}{|c|c|c|c|}
\hline $\begin{array}{l}\text { Oculus et structurae } \\
\text { pertinentes }\end{array}$ & $\begin{array}{l}\text { Eye and related } \\
\text { structures }\end{array}$ & $\begin{array}{l}\text { Olho e estruturas } \\
\text { pertinentes }\end{array}$ & $\begin{array}{l}\text { Olho e estruturas } \\
\text { pertinentes }\end{array}$ \\
\hline BULBUS OCULI & EYEBALL & BULBO DO OLHO & OLHO \\
\hline Polus anterior & Anterior pole & Pólo anterior & Pólo anterior \\
\hline Polus posterior & Posterior pole & Pólo posterior & Pólo posterior \\
\hline Equator & Equator & Equador & Equador \\
\hline Meridiani & Meridians & Meridianos & Meridianos \\
\hline Axis bulbi externus & External axis of eyeball & Eixo externo do bulbo & Eixo externo do olho \\
\hline Axis bulbi internus & Internal axis of eyeball & Eixo interno do bulbo & Eixo interno do olho \\
\hline Axis opticus & Optic axis & Eixo óptico & Eixo óptico \\
\hline Segmentum anterius & Anterior segment & Segmento anterior & Segmento anterior \\
\hline Segmentum posterius & Posterior segment & Segmento posterior & Segmento posterior \\
\hline Tunica fibrosa bulbi & Fibrous layer of eyeball & Túnica fibrosa do bulbo & Túnica fibrosa do olho \\
\hline Sclera & Sclera & Esclera & Esclera \\
\hline Sulcus sclerae & Sulcus sclerae & Sulco da esclera & Sulco da esclera \\
\hline Reticulum trabeculare & Trabecular tissue & Retículo trabecular & $\begin{array}{l}\text { Rede trabecular; } \\
\text { malha trabecular }\end{array}$ \\
\hline Pars corneoscleralis & Corneoscleral part & Parte corneoescleral & Parte corneoescleral \\
\hline Pars uvealis & Uveal part & Parte uveal & Parte uveal \\
\hline Calcar sclerae & Scleral spur & Esporão da esclera & Esporão escleral \\
\hline Sinus venosus sclerae & Scleral venous sinus & Seio venoso da esclera & Seio venoso da esclera \\
\hline Lamina episcleralis & Episcleral layer & Lâmina episcleral & Lâmina episcleral \\
\hline Substantia propria sclerae & Substantia propria & Substância própria da esclera & Substância própria da esclera \\
\hline Lamina fusca sclerae & Suprachoroid lamina & Lâmina fosca da esclera & Lâmina fosca da esclera \\
\hline Lamina cribrosa sclerae & Lamina cribrosa of sclera & Lâmina cribriforme da esclera & Lâmina crivosa da esclera \\
\hline Cornea & Cornea & Córnea & Córnea \\
\hline Anulus conjunctivae & Conjunctival ring & Anel da conjuntiva & Anel da conjuntiva \\
\hline Limbus corneae & $\begin{array}{l}\text { Corneoscleral junction; } \\
\text { Corneal limbus }\end{array}$ & Limbo da córnea & Limbo da córnea \\
\hline Vertex corneae & Corneal vertex & Vértice da córnea & Vértice da córnea \\
\hline Facies anterior & Anterior surface & Face anterior & Face anterior \\
\hline Facies posterior & Posterior surface & Face posterior & Face posterior \\
\hline Epithelium anterius & Corneal epithelium & Epitélio anterior & Epitélio \\
\hline Lamina limitans anterior & Anterior limiting lamina & Lâmina limitante anterior & Lâmina limitante anterior \\
\hline Substantia propria & Substantia propria & Substância própria & Substância própria \\
\hline Lamina limitans posterior & Posterior limiting lamina & Lâmina limitante posterior & Lâmina limitante posterior \\
\hline Epithelium posterius & Endothelium of anterior chamber & Epitélio posterior & Endotélio \\
\hline Tunica vasculosa bulbi & Vascular layer of eyeball & Túnica vascular do bulbo & Túnica vascular do olho \\
\hline Choroidea & Choroid & Corióide & Coróide \\
\hline Lamina suprachoroidea & Suprachoroid lamina & Lâmina supracorióide & Lâmina supracoroidea \\
\hline Spatium perichoroideum & Perichoroidal space & Espaço pericorióideo & Espaço pericoróideo \\
\hline Lamina vasculosa & Vascular lamina & Lâmina vascular & Lâmina vascular \\
\hline Lamina choroidocapillaris & Capillary lamina & Lâmina corióideocapilar & Lâmina coriocapilar \\
\hline Lamina basalis & Basal lamina & Lâmina basilar & Lâmina basal \\
\hline Vasa sanguinea choroideae & Choroid blood vessels & Vasos sangüíneos da corióide & Vasos sangüíneos da coróide \\
\hline Corpus ciliare & Ciliary body & Corpo ciliar & Corpo ciliar \\
\hline Corona ciliaris & Corona ciliaris & Coroa ciliar & Coroa ciliar \\
\hline Processus ciliares & Ciliary processes & Processos ciliares & Processos ciliares \\
\hline Plicae ciliares & Ciliary plicae & Pregas ciliares & Pregas ciliares \\
\hline Orbiculus ciliaris & Orbiculus ciliaris & Orbículo ciliar & Orbículo ciliar \\
\hline M. ciliaris & Ciliary muscle & M. ciliar & M. ciliar \\
\hline Fibrae meridionales & Meridional fibres & Fibras meridionais & Fibras meridionais \\
\hline Fibrae longitudinales & Longitudinal fibres & Fibras longitudinais & Fibras Iongitudinais \\
\hline Fibrae radiales & Radial fibres & Fibras radiais & Fibras radiais \\
\hline Fibrae circulares & Circular fibres & Fibras circulares & Fibras circulares \\
\hline Lamina basalis & Basal lamina & Lâmina basilar & Lâmina basal \\
\hline
\end{tabular}


Continuação

Quadro 1. Lista dos termos anatômicos referentes ao olho e estruturas pertinentes. As duas primeiras colunas apresentam os termos em latim e os de uso corrente em inglês, aprovados pelo FICAT e publicados conjuntamente ${ }^{(3)}$. A terceira coluna apresenta os termos em língua portuguesa aprovados pela SBA e reconhecidos pelo $\mathrm{FICAT}^{(4)}$. Na quarta coluna apresentamos uma lista com os termos de uso corrente

Iris

Margo pupillaris

Margo ciliaris

Facies anterior

Facies posterior

Anulus iridis major

Anulus iridis minor

Plicae iridis

Pupilla

M. sphincter pupillae

M. dilatator pupillae

Stroma iridis

Epithelium pigmentosum

Spatia anguli iridocornealis

Circulus arteriosus iridis major

Circulus arteriosus iridis minor

(Membrana pupillaris)

Tunica interna bulbi

Retina

Pars caeca retinae

Pars ciliaris retinae

Pars iridica retinae

Ora serrata

Pars optica retinae

Stratum pigmentosum

Stratum nervosum

Stratum segmentorum

externorum et internorum

Stratum limitans externum

Stratum nucleare externum

Stratum plexiforme externum

Stratum nucleare internum

Stratum plexiforme internum

Stratum ganglionicum

Stratum neurofibrarum

Stratum limitans internum

Discus nervi optici

Excavatio disci

Macula lutea

Fovea centralis

Foveola

Nervus opticus

Pars intracranialis

Pars canalis

Pars orbitalis

Pars intraocularis

Pars postlaminaris

Pars intralaminaris

Pars prelaminaris

Vagina externa

Vagina interna

Spatium intervaginale

subarachnoidale; spatium

leptomeningeum
Iris

Pupillary margin

Ciliary margin

Anterior surface

Posterior surface

Outer border of iris

Inner border of iris

Folds of iris

Pupil

Sphincter pupillae

Dilator pupillae

Stroma of iris

Pigmented epithelium

Spaces of iridocorneal angle

Major circulus arteriosus of iris

Minor circulus arteriosus of iris

(Pupillary membrane)

Inner layer of eyeball

Retina

Nonvisual retina

Ciliary part of retina

Iridial part of retina

Ora serrata

Optic part of retina

Pigmented layer

Neural layer

Layer of inner and outer

segments

Outer limiting layer

Outer nuclear layer

Outer plexiform layer

Inner nuclear layer

Inner plexiform layer

Ganglionic layer

Layer of nerve fibres

Inner limiting layer

Optic disc

Depression of optic disc;

Physiologcal cup

Macula

Fovea centralis

Foveola

\section{Optic nerve}

Intracranial part

Part in canal

Orbital part

Intraocular part

Postlaminar part

Intralaminar part

Prelaminar part

Outer sheath

Inner sheath

Subarachnoid space;

leptomeningeal space
Íris

Margem pupilar

Margem ciliar

Face anterior

Face posterior

Anel maior da íris

Anel menor da íris

Pregas da íris

Pupila

M. esfíncter da pupila

M. dilatador da pupila

Estroma da íris

Epitélio pigmentado

Espaços do ângulo iridocorneal

Círculo arterioso maior da íris

Círculo arterioso menor da íris

(Membrana pupilar)

Túnica interna do bulbo

Retina

Parte cega da retina

Parte ciliar da retina

Parte irídica da retina

Ora serrata

Parte óptica da retina

Estrato pigmentoso

Estrato nervoso

Estrato dos segmentos

externo e interno

Estrato limitante externo

Estrato nuclear externo

Estrato plexiforme externo

Estrato nuclear interno

Estrato plexiforme interno

Estrato ganglionar

Estrato das neurofibras

Estrato limitante interno

Disco do nervo óptico

Escavação do disco

Mácula lútea

Fóvea central

Fovéola

Nervo óptico

Parte intracraniana

Parte do canal

Parte orbital

Parte intra-ocular

Parte pós-laminar

Parte intralaminar

Parte pré-laminar

Bainha externa

Bainha interna

Espaço intervaginal

subaracnóideo; espaço

leptomeníngeo
Íris

Margem pupilar

Margem ciliar

Face anterior

Face posterior

Anel maior da íris

Anel menor da íris

Pregas da íris

Pupila

M. esfíncter da pupila

M. dilatador da pupila

Estroma da íris

Epitélio pigmentado

Espaços do ângulo iridocorneal

Círculo arterioso maior da íris

Círculo arterioso menor da íris (Membrana pupilar)

\section{Túnica interna do olho}

Retina

Parte cega da retina

Parte ciliar da retina

Parte irídica da retina

Ora serrata

Parte óptica da retina

Estrato pigmentado

Estrato nervoso

Camada dos segmentos

externo e interno

Camada limitante externa; membrana limitante externa Camada nuclear externa

Camada plexiforme externa

Camada nuclear interna

Camada plexiforme interna

Camada ganglionar

Camada de fibras nervosas

Camada limitante interna;

membrana limitante interna

Disco do nervo óptico

Escavação do disco

Mácula lútea

Fóvea central

Fovéola

Nervo óptico

Parte intracraniana

Parte intracanalicular

Parte orbitária

Parte intra-ocular

Parte pós-laminar

Parte intralaminar

Parte pré-laminar

Bainha externa

Bainha interna

Espaço intervaginal

subaracnóideo:

espaço leptomeníngeo 
Continuação

Quadro 1. Lista dos termos anatômicos referentes ao olho e estruturas pertinentes. As duas primeiras colunas apresentam os termos em latim e os de uso corrente em inglês, aprovados pelo FICAT e publicados conjuntamente ${ }^{(3)}$. A terceira coluna apresenta os termos em língua portuguesa aprovados pela SBA e reconhecidos pelo $\mathrm{FICAT}^{(4)}$. Na quarta coluna apresentamos uma lista com os termos de uso corrente na literatura oftalmológica nacional.

\section{Vasa sanguinea retinae}

A. centralis retinae,

pars intraocularis

Circulus vasculosus nervi optici

Arteriola temporalis retinae

superior

Arteriola temporalis retinae inferior

Arteriola nasalis retinae superior

Arteriola nasalis retinae inferior

Arteriola macularis superior

Arteriola macularis inferior

Arteriola macularis media

V. centralis retinae, pars

intraocularis

Venula temporalis retinae

superior

Venula temporalis retinae inferior

Venula nasalis retinae superior

Venula nasalis retinae inferior

Venula macularis superior

Venula macularis inferior

Venula macularis media

\section{Retinal blood vessels}

Central retinal artery,

intraocular part

Vascular circle of optic nerve

Superior temporal retinal arteriole

Inferior temporal retinal arteriole

Superior nasal retinal arteriole

Inferior nasal retinal arteriole

Superior macular arteriole

Inferior macular arteriole

Middle macular arteriole

Central retinal vein,

intraocular part

Superior temporal retinal venule

Inferior temporal retinal venule

Superior nasal retinal venule

Inferior nasal retinal venule

Superior macular venule

Inferior macular venule

Middle macular venule
Vasos sangüíneos da retina

A. central da retina,

parte intra-ocular

Círculo vascular do nervo óptico

Arteríola temporal superior da retina

Arteríola temporal inferior da retina

Arteríola nasal superior da retina

Arteríola nasal inferior da retina

Arteríola macular superior

Arteríola macular inferior

Arteríola macular média

V. central da retina, parte

intra-ocular

Vênula temporal superior da retina

Vênula temporal inferior da retina

Vênula nasal superior da retina

Vênula nasal inferior da retina

Vênula macular superior

Vênula macular inferior

Vênula macular média
Vasos sangüíneos da retina

A. central da retina, parte

intra-ocular

Círculo vascular do nervo óptico

Arteríola temporal

superior da retina

Arteríola temporal inferior da retina

Arteríola nasal superior da retina

Arteríola nasal inferior da retina

Arteríola macular superior

Arteríola macular inferior

Arteríola macular média

V. central da retina, parte intra-ocular

Vênula temporal superior da retina

Vênula temporal inferior da retina

Vênula nasal superior da retina

Vênula nasal inferior da retina

Vênula macular superior

Vênula macular inferior

Vênula macular média

\section{Lens}

Substantia lentis

Cortex lentis

Nucleus lentis

Fibrae lentis

Epithelium lentis

Capsula lentis

Polus anterior

Polus posterior

Facies anterior

Facies posterior

Axis

Equator

Radii

Zonula ciliaris

Fibrae zonulares

Spatia zonularia

\section{Camerae bulbi}

Humor aquosus

Camera anterior

Angulus iridocornealis

Camera posterior

Camera postrema;

Camera vitrea

Spatium retrozonulare

Corpus vitreum

(A. hyaloidea)

Canalis hyaloideus

Fossa hyaloidea

Membrana vitrea

Stroma vitreum

Humor vitreus

\section{Lens}

Lens substance

Cortex of lens

Lens fibres

Lens epithelium

Anterior pole

Posterior pole

Anterior surface

Posterior surface

Axis

Equator

Radii

Ciliary zonule

Zonular spaces
Nucleus of lens

Capsule of lens

Zonular fibres

\section{Lente}

Substância da lente

Córtex da lente

Núcleo da lente

Fibras da lente

Epitélio da lente

Cápsula da lente

Pólo anterior

Pólo posterior

Face anterior

Face posterior

Eixo

Equador

Raio

Zônula ciliar

Fibras zonulares

Espaços zonulares
Lente; Cristalino

Substância da lente

Córtex da lente

Núcleo da lente

Fibras da lente

Epitélio da lente

Cápsula da lente

Pólo anterior

Pólo posterior

Face anterior

Face posterior

Eixo

Equador

Raio

Zônula ciliar

Fibras zonulares

Espaços zonulares

Câmaras do bulbo do olho

Humor aquoso

Câmara anterior

Ângulo iridocorneal

Câmara posterior

Câmara póstrema

Postremal chamber;

Vitreous chamber

Retrozonular space

Vitreous body

(Hyaloid artery)

Hyaloid canal

Hyaloid fossa

Vitreous membrane

Vitreous stroma

Vitreous humor
Espaço retrozonular

Corpo vítreo

(A. hialóidea)

Canal hialóideo

Fossa hialóidea

Membrana vítrea

Estroma vítreo

Humor vítreo
Câmaras do olho

Humor aquoso

Câmara anterior

Ângulo iridocorneal

Câmara posterior

Câmara póstrema;

Câmara vítrea

Espaço retrozonular

Corpo vítreo

(A. hialóidea)

Canal hialóideo

Fossa hialóidea

Membrana vítrea

Estroma vítreo
Humor vítreo 
Continuação

Quadro 1. Lista dos termos anatômicos referentes ao olho e estruturas pertinentes. As duas primeiras colunas apresentam os termos em latim e os de uso corrente em inglês, aprovados pelo FICAT e publicados conjuntamente ${ }^{(3)}$. A terceira coluna apresenta os termos em língua portuguesa aprovados pela SBA e reconhecidos pelo $\mathrm{FICAT}^{(4)}$. Na quarta coluna apresentamos uma lista com os termos de uso corrente na literatura oftalmológica nacional.

Structurae oculi accessoriae
Periorbita
Septum orbitale
Vagina bulbi
Lig. suspensorium bulbi
Spatium episclerale
Corpus adiposum orbitae
Fasciae musculares
Musculi externi bulbi oculi

M. orbitalis

M. rectus superior

M. rectus inferior

$M$. rectus medialis

M. rectus lateralis

Lacertus musculi recti lateralis

Anulus tendineus communis

M. obliquus superior

Trochlea

Vagina tendinis musculi

obliqui superioris

M. obliquus inferior

M. levator palpebrae superioris

Lamina superficialis

Lamina profunda

\section{Supercilium}

\section{Palpebrae}

Palpebra superior

Palpebra inferior

Facies anterior palpebrae

Plica palpebronasalis

Facies posterior palpebrae

Rima palpebrarum

Commissura lateralis

palpebrarum

Commisura medialis

palpebrarum

Angulus oculi lateralis

Angulus oculi medialis

Limbus anterior palpebrae

Limbus posterior palpebrae

Cilia

Tarsus superior

Tarsus inferior

Lig. palpebrale laterale

Lig. palpebrale mediale

Glandulae tarsales

Glandulae ciliares

Glandulae sebaceae

M. tarsalis superior

M. tarsalis inferior

\section{Accessory visual structures}

\section{Periorbita}

Orbital septum

Fascial sheath of eyeball

Suspensory ligament of eyeball

Episcleral space

Retrobulbar fat; Orbital fat body

Muscular fascia

Extra-ocular muscles;

Extrinsic muscles of eyeball

Orbitalis; Orbital muscle

Superior rectus

Inferior rectus

Medial rectus

Lateral rectus

Check ligament of lateral

rectus muscle

Common tendinous ring;

Common anular tendon

Superior oblique

Trochlea

Tendinous sheath of superior oblique

Inferior oblique

Levator palpebrae superioris

Superficial layer

$$
\text { Deep layer }
$$

\section{Eyebrow}

\section{Eyelids}

Superior eyelid; Upper eyelid Inferior eyelid; lower eyelid

Anterior surface of eyelid

Palpebronasal fold;

medial canthic fold

Posterior surface of eyelid

Palpebral fissure

Lateral palpebral comissure

Medial palpebral comissure

Lateral angle of eye

Medial angle of eye

Anterior palpebral margin

Posterior palpebral margin

Eyelash

Superior tarsus

Inferior tarsus

Lateral palpebral ligament

Medial palpebral ligament

Tarsal glands

Ciliary glands

Sebaceous glands

Superior tarsal muscle

Inferior tarsal muscle
Estruturas oculares

acessórias

Periórbita

Septo orbital

Bainha do bulbo

Lig. suspensor do bulbo

Espaço episcleral

Corpo adiposo da órbita

Fáscias musculares

Músculos extrínsecos

do bulbo

M. orbital

M. reto superior

M. reto inferior

M. reto medial

M. reto lateral

Prolongamento do músculo reto lateral

Anel tendíneo comum

M. oblíquo superior

Tróclea

Bainha tendínea do $\mathrm{m}$. oblíquo superior

M. oblíquo inferior

M. levantador da pálpebra

superior

Lâmina superficial

Lâmina profunda

Supercílio

Pálpebras

Pálpebra superior

Pálpebra inferior

Face anterior da pálpebra

Prega palpebronasal

Face posterior da pálpebra

Rima das pálpebras

Comissura lateral das pálpebras

Comissura medial das pálpebras

Ângulo lateral do olho

Ângulo medial do olho

Limbo anterior da pálpebra

Limbo posterior da pálpebra

Cílios

Tarso superior

Tarso inferior

Lig. palpebral lateral

Lig. palpebral medial

Glândulas tarsais

Glândulas ciliares

Glândulas sebáceas

M. tarsal superior

M. tarsal inferior
Estruturas oculares

acessórias

Periórbita

Septo orbitário

Bainha do olho; fáscia bulbar

Lig. suspensor do olho

Espaço episcleral

Corpo adiposo da órbita

Fáscias musculares

Músculos extrínsecos do olho

M. orbitário

M. reto superior

M. reto inferior

M. reto medial

M. reto lateral

Prolongamento do

músculo reto lateral

Anel tendinoso comum

M. oblíquo superior

Tróclea

Bainha tendinosa do m. oblíquo superior

M. oblíquo inferior

M. levantador da pálpebra superior

Lâmina superficial

Lâmina profunda

Supercílio

Pálpebras

Pálpebra superior

Pálpebra inferior

Face anterior da pálpebra

Prega palpebronasal

Face posterior da pálpebra

Rima das pálpebras

Comissura lateral das pálpebras

Comissura medial das pálpebras

Ângulo lateral do olho

Ângulo medial do olho

Limbo anterior da pálpebra

Limbo posterior da pálpebra

Cílios

Tarso superior

Tarso inferior

Lig. palpebral lateral

Lig. palpebral medial

Glândulas tarsais

Glândulas ciliares

Glândulas sebáceas

M. tarsal superior

M. tarsal inferior 


\section{Continuação}

Quadro 1. Lista dos termos anatômicos referentes ao olho e estruturas pertinentes. As duas primeiras colunas apresentam os termos em latim e os de uso corrente em inglês, aprovados pelo FICAT e publicados conjuntamente ${ }^{(3)}$. A terceira coluna apresenta os termos em língua portuguesa aprovados pela SBA e reconhecidos pelo $\mathrm{FICAT}^{(4)}$. Na quarta coluna apresentamos uma lista com os termos de uso corrente na literatura oftalmológica nacional.

\section{Tunica conjunctiva}

Plica semilunaris

Caruncula lacrimalis

Tunica conjunctiva bulbi

Tunica conjunctiva palpebrarum

Fornix conjunctivae superior

Fornix conjunctivae inferior

Saccus conjunctivalis

Glandulae conjunctivales

Apparatus lacrimalis

Glandula lacrimalis

Pars orbitalis

Pars palpebralis

Ductuli excretorii

(Glandulae lacrimales

accessoriae)

Rivus lacrimalis

Lacus lacrimalis

Papilla lacrimalis

Punctum lacrimale

Canaliculus lacrimalis

Ampulla canaliculi lacrimalis

Saccus lacrimalis

Fornix sacci lacrimalis

Ductus nasolacrimalis

\section{Conjunctiva}

Plica semilunaris

Lacrimal caruncle

Bulbar conjunctiva

Palpebral conjunctiva

Superior conjunctival fornix Inferior conjunctival fornix

Conjunctival sac

Conjunctival glands

Lacrimal apparatus

Lacrimal gland

Orbital part

Palpebral part

Excretory ducts

(Accessory lacrimal glands)

Lacrimal pathway

Lacus lacrimalis; Lacrimal lake

Lacrimal papilla

Lacrimal punctum

Lacrimal canaliculus

Ampulla of lacrimal canaliculus

Lacrimal sac

Fornix of lacrimal sac

Nasolacrimal duct
Túnica conjuntiva

Prega semilunar

Carúncula lacrimal

Túnica conjuntiva do bulbo

Túnica conjuntiva da pálpebra

Fórnice superior da conjuntiva Fórnice inferior da conjuntiva

Saco da conjuntiva

Glândulas conjuntivais

Aparelho lacrimal

Glândula lacrimal

Parte orbital

Parte palpebral

Dúctulos excretores

(Glândulas lacrimais

acessórias)

Rego lacrimal

Lago lacrimal

Papila lacrimal

Ponto lacrimal

Canalículo lacrimal

Ampola do canalículo lacrimal

Saco lacrimal

Fórnice do saco lacrimal

Ducto lacrimonasal
Túnica conjuntiva

Prega semilunar

Carúncula lacrimal

Túnica conjuntiva do olho; conjuntiva bulbar

Túnica conjuntiva da pálpebra; conjuntiva palpebral

Fórnice superior da conjuntiva Fórnice inferior da conjuntiva

Saco conjuntival

Glândulas conjuntivais

Aparelho lacrimal

Glândula lacrimal

Parte orbital

Parte palpebral

Dúctos excretores

(Glândulas lacrimais

acessórias)

Rio lacrimal

Lago lacrimal

Papila lacrimal

Ponto lacrimal

Canalículo lacrimal

Ampola do canalículo lacrimal

Saco lacrimal

Fórnice do saco lacrimal

Ducto lacrimonasal;

ducto nasolacrimal

Prega lacrimal

Plica lacrimalis

Lacrimal fold

Prega lacrimal

( ): Utilizado para termos de estruturas anatômicas inconstantes; Coluna 1 - abreviaturas utilizadas: A.: Arteria; Lig.: Ligamentum; M.: Musculus; V.: Vena; Colunas 3 e 4 - abreviaturas utilizadas: A.: Artéria; Lig.: Ligamento; M.: Músculo; V.: Veia

Quadro 2. Lista de epônimos relacionados às estruturas anatômicas do olho e seus correspondentes de acordo com a Terminologia Anatômica

Bowman, membrana de - Lâmina limitante anterior.

Bruch, membrana de - Lâmina basilar da corióide.

Brücke, músculo de - Fibras meridionais do M. ciliar.

Descemet, membrana de - Lâmina limitante posterior.

Fontana, espaços de - Espaços do ângulo iridocorneal.

Gratiolet, radiação de - Radiação óptica.

Haller, camada de - Lâmina vascular da corióide.

Hannover, canal/espaço de - Espaços zonulares.

Hasner, valva de - Prega lacrimal.

Horner, músculo de - Parte profunda (parte palp. M. orbicular).

Krause, glândulas de - Glândula lacrimal acessória.

Lockwood, ligamento de - Lig. suspensor do bulbo do olho.

Maier, seio de - Fórnice do saco lacrimal.
Meibômio, glândulas de - Glândulas tarsais.

Moll, glândula de - Glândulas ciliares.

Müller, músculo de - Fibras circulares do M. ciliar.

Müller, músculo de - Músculo orbital.

Petit, canal/espaços de - Espaços zonulares.

Sattler, camada de - Lâmina vascular da corióide.

Schlemm, canal de - Seio venoso da esclera.

Stilling, canal de - Câmara póstrema.

Tenon, cápsula de - Bainha do bulbo do olho.

Whitnall, tubérculo de - (Tubérculo marginal - órbita).

Wolfring, glândulas de - (Glândulas lacrimais acessórias).

Zeiss, glândulas de - Glândulas sebáceas da pálpebra.

Zinn, zônula de - Zônula ciliar.

\section{VOCABULÁRIO ETIMOLÓGICO DE TERMOS} ANATÔMICOS EM OFTALMOLOGIA

Anatomia é palavra grega que significa cortar de permeio, separar em partes; origina-se de aná (prefixo que, entre outros sentidos, tem os de sobre, para cima) e tomia (= corte). É sinônimo da palavra latina dissecação, de dis (= separação, divisão), e secare (= cortar).
Anatomia é, pois, o estudo das partes de seres organizados.

Sendo os seres organizados vegetais e animais, teremos, então, uma Anatomia Vegetal ou Fitoanatomia, do grego phytos (= planta) e uma Anatomia Animal, Zooanatomia, do grego zôo (= animal).

$\mathrm{Na} Z$ Zooanatomia, que é o que nos interessa, estudaremos a Androanatomia, ou Antomia Humana, de anér, andrós (= homem), ou Antropoanatomia, de antropos, ántropou (= homem). 


\begin{tabular}{|c|c|c|}
\hline Descritor Português & Sinônimos Português & Descritor Inglês \\
\hline Olho & & Eye \\
\hline Segmento anterior do olho & & Anterior eye segment \\
\hline Câmara anterior & & Anterior chamber \\
\hline Humor aquoso & Rubor aquoso; Líquido intra-ocular & Aqueous Humor \\
\hline Endotélio da córnea & $\begin{array}{l}\text { Epitélio da câmara anterior; } \\
\text { Endotélio da Câmara anterior }\end{array}$ & Endothelium, Corneal \\
\hline Córnea & & Cornea \\
\hline Epitélio da córnea & & Epithelium, Corneal \\
\hline Membrana de Bowman & & Bowman Membrane \\
\hline Estroma corneano & Estroma da córnea & Corneal Stroma \\
\hline Lâmina limitante posterior da córnea & Lâmina limitante posterior; Membrana de Descemet & Descemet Membrane \\
\hline Endotélio da córnea & $\begin{array}{l}\text { Epitélio da câmara anterior; } \\
\text { Endotélio da câmara anterior }\end{array}$ & Endothelium, Corneal \\
\hline Limbo da córnea & $\begin{array}{l}\text { Limbo corneano; Junção corneoescleral; } \\
\text { Limbo esclerocorneano }\end{array}$ & Limbus Corneae \\
\hline Malha trabecular & & Trabecular Meshwork \\
\hline Conjuntiva & & Conjunctiva \\
\hline Pálpebras & & Eyelids \\
\hline Pestanas & & Eyelashes \\
\hline Glândulas meibomianas & & Meibomian Glands \\
\hline Aparelho lacrimal & Glândula lacrimal & Lacrimal Apparatus \\
\hline Ducto nasolacrimal & & Nasolacrimal Duct \\
\hline Cristalino & $\begin{array}{l}\text { Cristalino do olho; Lente do olho; } \\
\text { Lente (cristalino); Lente ocular }\end{array}$ & Lens, Crystalline \\
\hline Cápsula do cristalino & & Lens Capsule, Crystalline \\
\hline Córtex do cristalino & & Lens Cortex, Crystalline \\
\hline Núcleo do cristalino & & Lens Nucleus, Crystalline \\
\hline Músculos oculomotores & & Oculomotor Muscles \\
\hline Epitélio pigmentado ocular & Epitélio pigmentado da retina & Pigment Epithelium of Eye \\
\hline Retina & & Retina \\
\hline Células amácrinas & & Amacrine Cells \\
\hline Barreira hemato-retiniana & $\begin{array}{l}\text { Barreira sangue-retina; Barreira retina-sangue; } \\
\text { Barreira retino-hemática; Barreira retinohemática }\end{array}$ & Blood-Retinal Barrier \\
\hline Fundo de olho & & Fundus Oculi \\
\hline Macula lutea & & Macula Lutea \\
\hline Fóvea central & & Fovea Centralis \\
\hline Disco óptico & $\begin{array}{l}\text { Mancha cega; Cabeça do nervo óptico; } \\
\text { Papila óptica }\end{array}$ & Optic Disc \\
\hline Fotorreceptores & Fotorreceptores retinianos & Photoreceptors \\
\hline Fotorreceptores de invertebrados & & Photoreceptors, Invertebrate \\
\hline Fotorreceptores de vertebrados & Bastonetes e cones & Photoreceptors, Vertebrate \\
\hline Cones (retina) & Cones fotorreceptores & Cones (Retina) \\
\hline Bastonetes (retina) & Bastonetes fotorreceptores & Rods (Retina) \\
\hline Segmentos externos do bastonete & & Rod Outer Segments \\
\hline Células bipolares da retina & Células bipolares retinianas & Retinal Bipolar Cells \\
\hline Células do gânglio retiniano & $\begin{array}{l}\text { Células ganglionares retinianas; } \\
\text { Células ganglionares da retina }\end{array}$ & Retinal Ganglion Cells \\
\hline Células horizontais da retina & Células horizontais retinianas & Retinal Horizontal Cells \\
\hline Esclera & & Sclera \\
\hline Úvea & & Uvea \\
\hline Barreira hemato-aquosa & $\begin{array}{l}\text { Barreira aquoso-hemática; Barreira aquoso-sangue; } \\
\text { Barreira aquosohemática; Barreira aquoso-sanguínea }\end{array}$ & Blood-Aqueous Barrier \\
\hline Coróide & Corióide & Choroid \\
\hline Membrana de Bruch & $\begin{array}{l}\text { Complexo basal coreorretiniano; } \\
\text { Lâmina basal da coróide. }\end{array}$ & Bruch Membrane \\
\hline Corpo ciliar & & Ciliary Body \\
\hline Íris & & Iris \\
\hline Pupila & & Pupil \\
\hline Corpo vítreo & Humor vítreo & Vitreous Body \\
\hline Fonte: Biblioteca Virtual em Saúde - Descritores & èncias da Saúde - http://decs.bvs.br & \\
\hline
\end{tabular}


A Anatomia Descritiva estuda os ossos (osteologia), de ostéon (= osso), as articulações (artrologia), de árthros (= articulação), os músculos (miologia), de mys, myós (= músculo), a circulação (angiologia), de ángion (= vaso), o sistema nervoso (neurologia), de néuron (= nervo), as vísceras (splancnologia), de splánchnon (= víscera).

A “Anatomia Geral" ou a "Histologia", de histós (= tecido), estuda as partes elementares dos tecidos. Fazem-se cortes delicados, e o estudo se realiza com o auxílio do microscópio.

\section{A}

Abdução Do lat. $a b$ (= afastamento, ponto de partida, separação, privação, para longe) e ductor, de ducere (= conduzir).

Adito Do lat. aditus, us aditus (= entrada ou acesso a um lugar; ponto de acesso a uma cavidade).

Adução Do lat. adductio, adductionis, de adducere $a d+$ duccere (= puxar para si).

Aferente Do lat. afferens, afferentis de ad, direção para, assimilado em af, e ferens, ferentis (= que vem vindo).

Alça Do lat. ansa, ansae (= asa de qualquer objeto). Em gr., labé, labés (= alça, punho).

Também pode ser derivado regressivo de alçar.

Angiologia Do gr. angeiologia, de angéion (= vaso), logia, de log (= ciência).

Aquoso Do lat. aquosus, de aqua, aquae (= água). Em gr., hydatódes (= semelhante à água, aquoso, que vem de hydor, hydatos (= água).

Aracnóide Do gr. arachnolidés (= semelhante à teia de aranha), de arachnés, arachnon (= aranha) e eidos (óidos) (= semelhante).

Artéria Do gr. artéria, arterías, de aér (= ar), e terein (=manter,conduzir), (= canal de ar para respiração), pelo lat. arteria, arteriae. Pelo fato de ficarem vazias após a morte, os antigos as relacionaram a dutos de ar. Os brônquios e a traquéia também eram chamados de artérias.

Asa 1. Do lat. ansa (= ansae, cabo por onde se pega em alguma coisa). 2. De ala, alae (= membro empenado das aves). Em gr., ptéryx, ptérigos (= asa ou objeto semelhante à asa).

Assoalho Em gr., katástroma, kaktastrómatos, e saniou soalho dómatos (= superfície inferior de uma cavidade).

\section{B}

Bainha
Buraco

Do lat. foramen, foraminis (= buraco, abertura). Em gr., trema, trématos (= orifício) $e$ trypa, trypes (= buraco).

Na nomenclatura anatômica moderna, emprega-se forame preferentemente a buraco.

\section{C}

Cabeça Do lat. caput, caputis (Em gr., Kephalé, kephalés, de onde cefaléia)

Canal Do lat. canalis (= cano, tubo). Em gr., solén, solénos (= canal, tubo, ranhura).

Canalículo Do lat. canaliculus, canaliculi, diminutivo de canalis (= cano pequeno, estreito; canudo; tubozinho).

Cápsula Do lat. capsula, capsulae, diminutivo de capsa, capsae (= caixinha, cofrezinho, cápsula).

Carúncula Do lat. caruncula, carunculae, diminutivo de caros, carnis (= pouca carne, um bocadinho de carne).

Cavernoso Do lat. cavernosus, $a$, um (= de caverna. Que encerra pequenas cavidades, pequenas cavernas, ou que é um tecido vascular esponjoso).

Cerebral Do francês cerebral, que veio do lat. cerebralis, de cerebrum, cerebri.

Ciliar Do lat. cilium, ii (= a beira da capela do olho, (corpo ciliar) da pálpebra. Ciliar = relativo aos cílios ).

Concha Do gr. kónche, kónches (= casa, concavidade), pelo lat. concha, conchae.

Conjuntiva Do lat. conjunctivus, $a$, um, de conjungere (= que serve para ligar, unir).

Córnea Do lat. corneus, $a$, um (= consistência de corno ou chifre; semelhante a chifre).

Crânio Do gr. kranion (= crânio, cabeça), pelo lat. cranion (= parte da cabeça).

Cristalino Do gr. krystállinos, de krystallos (= vidro, gelo, cristal), pelo lat. crystallinus, a um (= de cristal).

\section{D}

Dácrio Do gr. dákryon (= lágrima).

Dental Do lat. dentalis, $e$ (= relativo ou pertencente aos dentes. O mesmo que dentário), pelo francês dental.

Díploe Do gr. díploe, díploes (= idéia de dois, coisa dupla).

Dura-máter Do lat. dura, durae (= firme, forte) e mater, matris (=mãe).

\section{$\mathbf{E}$}

Eferente

Do lat. efferens, efferentis (= o que leva para fora).

Encéfalo Do gr. enképhalos, de en (= dentro) e kephalé (= cérebro, o que está na cabeça). ceber). 
Endotélio Do gr. Endon (= dentro) e thele (= mamilo) Embora não haja mamilos nos endotélios, a origem foi a analogia com epitélio.

Epitélio Do gr.epi (= por cima) e thele (= mamilo). Primitivamente aplicava-se só às camadas celulares que cobriam as papilas da língua, depois estendeu-se para outras superfícies sem papilas, inclusive a pele.

Esclerótica Do gr. sklerótes, sklerótetos (= segurança, dureza). Hoje, em oftalmologia, é chamada de esclera.

Esfenóide Do gr. sphén, sphénos (= cunha ou ponta) e óide, ou eídos (= em forma de, ou semelhante a).

Esfíncter Do gr. sphinkter, sphinktéros (= o que aperta, laço). De sphíngein (= apertar).

Estroma Do gr; stroma (= o que se estende, que cobre). Etmóide Do gr. ethmos (= peneira, crivo) e oide, de eidos (= semelhante a).

\section{F}

Face Do lat.facies, faciei (= rosto, cara, semblante).

Fáscia Do lat.fascia, fasciae (= faixa, atadura).

Fissura Do lat.fissura, fissurae, de findere (= rachar, fender; fenda, rachadura).

Forame Do lat. foramen, foraminis (= buraco, cova, abertura).

Fossa Do lat.fossa, fossae, de fodere (= cavar, escavação, cova).

Frontal Do lat. frontalis (= que pertence à fronte ou à frente).

\section{G}

Gânglio Do gr. gánglion, pelo lat. ganglion, ganglii (= tumor, inchaço, pequenos corpos de forma e estruturas variáveis, de que se distinguem duas espécies: gânglios linfáticos (linfonódulos) e os gânglios nervosos.

Geniculado Do lat. geniculatus (= dobrado em forma de geniculum; joelho).

Glândula Do lat. glandula, glandulae (= diminutivo de glans, glandis (= bolota, glande)). Em gr. adén, adenos (= glande).

Glia Do gr. glia (= cola).

\section{H}

Humor Do lat. humor, humoris. Em gr. chymós, chymoü (= suco, líquido, fluido).

\section{I}

Íris Do gr. iris, iridos, pelo lat. iris, iridis (= arco-íris).

$\mathbf{L}$

Lágrima Do lat. lacrima, lacrimae. Em gr., dácryon (= lágrima; gota de seiva).
Lâmina

Lateral

Lente

Ligamento

Limbo

Mácula

Margem

Maxila

Maxilar

Medial

Membrana

Meninge

Mental

Músculo

Nervo

Neuroglia

Oblíquo

Oftálmico

Óptico

Orbicular

Órbita
Do lat. lamina, laminae. Em gr., elasmós, ou (= pedaço muito fino, folha, chapa, lâmina). Do lat. Lateralis (= afastado do plano mediano ou da linha média de um corpo ou estrutura; relativo a lado).

Do lat. lens, lentis (= lentilha - usado devido à semelhança de forma entre as lentes de vidro e aquele grão vegetal).

Do lat. ligamentum, $i$ (= ligadura, atadura). Do lat. limbus, $i$ (= borda ou margem; orla).

\section{M}

Do lat. macula, maculae (= mancha, mácula). Do lat. margo, marginis (= beira, borda, margem).

Do lat. maxilla, maxillae (= queixada, queixo). Do lat. maxillaris, maxilare. Em gr., gnátos, gnáton (= maxilar, queixal).

Do lat. mediale, medialis (= próximo do plano mediano ou da linha média de um corpo ou estrutura; relativo ao meio)

Do lat. membrana, membranae (= película, membrana).

Do gr. menix, meningos por intermédio do lat. meninge (= membrana muito fina; meninge).

Do lat. mentum, menti (quando se refere ao queixo); do lat. mens, mentis (quando se refere ao espírito).

Do lat. musculus, diminutivo de mus, muris (= camundongo). Talvez porque o tamanho e formato do músculo bíceps braquial tenham sido considerados semelhantes aos de um pequeno rato se movimentando sob a pele. A mesma analogia acontece em grego: mys (=rato), origem do prefixo "mio".

\section{$\mathbf{N}$}

Do lat. nervus, nervi (= nervo, corda, tendão). Do gr. neuron $(=$ nervo) e glia $(=$ cola $)$.

\section{$\mathbf{O}$}

Do lat. obliquus, a, um (= oblíquo, curvo, torto; que está de esguelha). Em gr., loxós (= direção oblíqua, curvo).

Do gr. ophtalmikós (= relativo aos olhos, ocular).

Do gr. optikós (= relativo à vista, visual); optormai (= eu vejo).

Do lat. orbicularis, e, derivado de orbis, orbis (= toda figura circular, anel, rodinha). Em Gr. kiclikós (= circular, redondo).

Do lat. orbita, orbitae, de orbis, orbis (= toda figura esférica ou circular).

Em gr. perifora, es (= 1. cavidade do olho; 2. percurso de um planeta).

Orbitário (= que tem relação com a órbita). 
Osteologia Do gr. osteologia de osteon (= osso) + logia (= ciência, estudo).

Ótico Do gr. otikós, de oûs, otós (= relativo ao ouvido).

\section{$\mathbf{P}$}

Palatino

Do lat. palato + ino $(=$ que se refere ao palato).

Pálpebra Do lat. palpebra, palpebrae, de palpare (Relaciona-se com tatear, tocar, aplacar, acalmar; para proteger e lubrificar o olho).

Papila Do lat. papilla, papillae (são pequenas eminências mais ou menos salientes, cônicas, que se elevam de uma superfície).

Pele Do lat. pellis, pellis (= cútis, couro). Em gr. dérma, dermatos.

Periósteo Do gr.periósteon, de peri (= em volta de, em torno de) e ostéon (= osso).

Pia-máter Do lat. pia (= piedosa) e mater $(=$ mãe).

Pupila Do lat. pupilla, pupillae, diminutivo de pupa, pupae (= menina). Em gr., kóre, kóres (=menina).

\section{Q}

Quiasma Do gr. chíasma, chiásmatos (= disposição em forma de cruz).

\section{$\mathbf{R}$}

Raiz Do lat. radix, radicis (= raiz). Em gr., riza, rizes (Figurado: base, fundamento, fonte, origem).

Ramo Do lat. ramus, rami. Em gr. ózos, ózon e kládos, kládon (= ramo).

Retina Do lat. retina, retinae, de rete, retis (= teia ou rede).

\section{$\mathbf{S}$}

Septo Do lat. saeptum, septi (= parede, muro). Em gr., diaphragma, diaphrágmatos (= separação).

Supercílio Do lat. supercillium, supercilli, de super (= acima) e cilium, cillii (= pestana superior, sobrancelha).

\section{$\mathbf{T}$}

Tróclea Do gr., trochalía, trochalías (= polia, molinete); pelo lat. trochlea, trochleae (= guindaste, roldana, polé).

Túnica Do lat. tunica, tunicae (= vestido). Em gr., chitón, chitonós (= vestimenta de baixo, túnica). Toda membrana que forma ou concorre para formar paredes de um órgão.

\section{$\mathbf{U}$}

Unguis Do lat. unguis, unguis (= unha).

Nome do osso lacrimal, assim chamado pelo seu feitio de unha.

Úvea

Do inglês uvea. Em gr., chorioeidés (= semelhante a uma membrana, revestimento), de chorión (= membrana).

V

Veia

Vórtex

Zigomático

Do gr. zugoma + ataxo (= armação, peça de sustentação).

Do lat. vena, venae. (= vaso de sangue) Do gr. Flebos. (origem do termo flebite).

Do lat. vortex, vorticis ( = turbilhão, redemoinho). Em gr. strómbos, strómbon (= turbilhão).

\section{$\mathbf{Z}$}

\section{ABSTRACT}

The purposes of this article are: to inform ophthalmologists about the differences between the English and Portuguese list of equivalent terms for eye structures, approved by the $\mathrm{Fe}$ derative International Committee on Anatomical Terminology; to present the anatomical terms included in the list of medical subject headings published by the United States National Library of Medicine and translated by the Regional Library of Medicine (BIREME); propose a list of Portuguese terms of common usage by ophthalmologists.

Keywords: Terminology; Anatomy; Eye; Ophthalmology; Medical subject headings; Eponyms; History of medicine.

\section{REFERÊNCIAS}

1. Atta HM. Edwin Smith Surgical Papyrus: the oldest known surgical treatise. Am Surg. 1999;65(12):1190-2.

2. Encyclopædia Britannica Online. Anatomical nomenclature. [Internet]. London: Encyclopaedia Brittanica. [cited 2007 June 8]. Available from: http:// www.britannica.com/eb/article-284/anatomy

3. Federative International Committee on Anatomical Terminology. Terminologia Anatomica: International Anatomical Terminology. Stuttgart: Thieme; 1998

4. Sociedade Brasileira de Anatomia. Comissão Federativa da Terminologia Anatômica, Terminologia Anatômica Internacional. São Paulo: Manole; 2001.

5. Abib FC, Oréfice F. Terminologia anatômica utilizada em oftalmologia. Arq Bras Oftalmol. 2005;68(2):273-6.

6. Lamy R, Pecego MG, Netto CF, Lima PH, Dantas AM. Avaliação do conhecimento da terminologia anatômica pelos oftalmologistas. Arq Bras Oftalmol. 2007;70(4 Supl):46-7.

7. Dantas AM. Anatomia funcional do olho e seus anexos. Rio de Janeiro: Colina; 2002.

8. Oréfice F, Bonfioli AA, Boratto LM. Biomicroscopia e gonioscopia: texto e atlas. $2^{\underline{a}}$ ed; Rio de Janeiro: Cultura Médica; 2001.

9. Belfort Jr R, Kara-José N. Córnea: clínica - cirúrgica. São Paulo: Roca; 1997.

10. Rodrigues ML, Dantas AM. Oftalmologia clínica. Rio de Janeiro: Cultura Médica; 2001.

11. Alves AA. Refração. $5^{\mathfrak{a}}$ ed. Rio de Janeiro: Cultura Médica; 2005.

12. Moreira H, Moreira SM, Moreira LB. Lentes de contato. $3^{\underline{a}}$ ed. Rio de Janeiro: Cultura Médica; 2004.

13. Yamane R. Semiologia ocular. $2^{\underline{a}}$ ed. Rio de Janeiro: Cultura Médica; 2003.

14. Moreira Jr CA, Freitas D, Kikuta HS. Trauma ocular. Rio de Janeiro: Cultura Médica; 2000.

15. Oréfice F. Uveite: clínica e cirúrgica: texto e atlas. $2^{\underline{a}}$ ed. Rio de Janeiro: Cultura Médica; 2005.

16. Dias JFP, Almeida HG. Glaucoma. $2^{\underline{a}}$ ed. Rio de Janeiro: Cultura Médica; 2000.

17. Rezende F. Cirurgia de catarata. Rio de Janeiro: Cultura Médica; 2002. 
18. Dantas AM, Monteiro ML. Doenças da órbita. Rio de Janeiro: Cultura Médica; 2002.

19. Abreu G. Ultra-sonografia ocular: atlas e texto. 3a ed. Rio de Janeiro: Cultura Médica; 2002.

20. Padilha M. Catarata. Rio de Janeiro: Cultura Médica; 2003.

21. Souza-Dias CR, Almeida HC. Estrabismo. 3a ed. São Paulo: Roca; 1998

22. Hofling-Lima AL, Melamed J, Calixto N. Terapêutica clínica ocular. São Paulo: Roca; 1995.

23. Soares EJ, Moura EM, Gonçalves JO. Cirurgia plástica ocular. São Paulo: Roca; 1997.

24. Abujamra S, Ávila M, Barsante C, Farah ME, Gonçalves JO, Lavinsky J, et al. Retina e vítreo: clínica e cirurgia. São Paulo: Roca; 2000

25. Kara-José N, Almeida GV, organizadores. Senilidade ocular. São Paulo: Roca; 2001

26. Alves MR, Chamon W, Nosé W, editores. Cirurgia refrativa. Rio de Janeiro: Cultura Médica; 2003.

27. Piatto VB, Batigália F, Neves AP. Terminologia médica e o uso de epônimos. HB Científica 2000;7(3):183-8.

28. Whitmore I. Terminologia anatomica: new terminology for the new anatomist. Anat Rec. 1999;257(2):50-3.
29. Coelho PJ. Vocabulário de termos anatômicos. Juiz de Fora: Universidade Federal de Juiz de Fora; 1967.

30. Feneis H. Dicionário ilustrado de anatomia. $4^{\underline{a}}$ ed. Rio de Janeiro: Cultura Médica; 1976

31. Mitchell GA. Nomina anatomica. $3^{\text {th }}$ ed. Amsterdam: Excerpta Medica Foundation; 1968.

32. Renard G, Lemasson C, Saraux H. Anatomie de l'oeil et de ses annexes. Paris: Masson; 1965.

33. Dorland NW. Dorland's illustrated medical dictionary. Philadelphia: W.B. Saunders; 2007.

34. Heckler E, Back S, Massing E. Dicionário morfológico da língua portuguesa. Rio Grande do Sul: Unisinos; 1984.

35. Houaiss A. Dicionário Houaiss da língua portuguesa. Rio de Janeiro: Objetiva; 2001.

36. Machado JP. Dicionário onomástico etimológico da língua portuguesa. Lisboa: Livros Horizonte; 1987

37. Saraiva FRS. Novíssimo dicionário latino-português. $10^{\underline{a}}$ ed. Rio de Janeiro: Garnier; 1993.

38. Pereira I. Dicionário grego-português e português-grego. Braga, Portugal: Liv. Apostolado da Imprensa; 1998. 